\title{
Development and Characterization of a Novel Vesicular System for Topical Delivery: An Opportunity for Dimethylsulfoxide
}

Sandra Simões, Tiago Mendes, Carla Eleutério, Pedro Pinto and Andreia Ascenso*

iMed.ULisboa, Faculdade de Farmácia, Universidade de Lisboa, Lisbon, Portugal

\begin{abstract}
Current use of Dimethylsulfoxide (DMSO) in dermatology and in topical formulations is scarce despite its potential as a skin penetration enhancer and as a pharmacological active itself. The aim of this work is to develop and characterize a new type of semisolid formulation of Soybean Phosphatidylcholine (SPC) - DMSO deformable nanovesicles for topical application. Tretinoin is used in this work as a model substance. The formulation presents a non-newtonian pseudoplastic behaviour and a pH compatible with the skin. Stability-on-storage at different temperatures indicate an excellent physical and chemical stability and SPC-DMSO vesicles show high deformability when forced to pass through pores much smaller than their own size. These new nanovesicles show a high Tretinoin yield. Calorimetric analysis indicates a possible interaction between Tretinoin and DMSO in the lipid vesicle bilayer and vesicles formulation exhibit low cytotoxic effect below $1.25 \%$ SPC. Moreover, these vesicles once applied on the skin do not cause irritation. This study presents a new opportunity in dermal delivery for a controversial molecule such as DMSO. SPC-DMSO vesicles have plenty of advantages for topical application and future studies should confirm their superiority as drug carriers for topical application as well as for clinical evaluations in managing skin disorders.
\end{abstract}
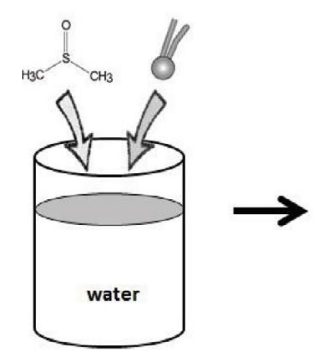
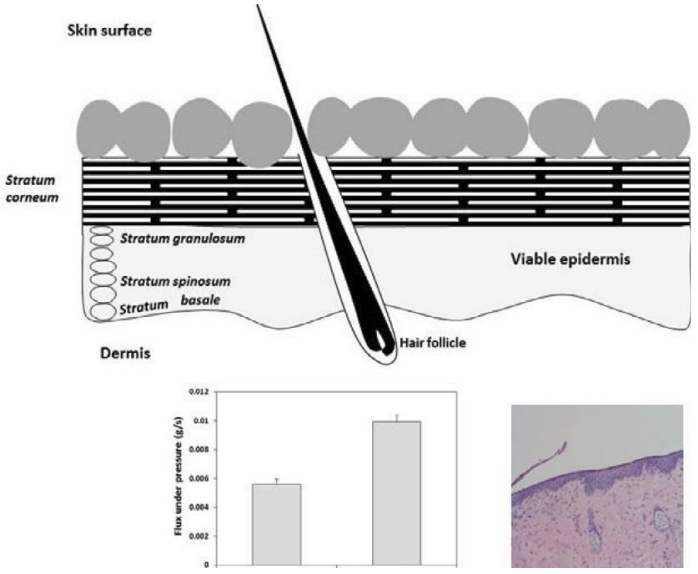

Keywords: Dimethylsulfoxide; Tretinoin; Soybean phosphatidylcholine; Nanovesicles

\section{Introduction}

Dimethylsulfoxide (DMSO) is a polar aprotic solvent miscible with water and able to dissolve polar and nonpolar small molecules. It has been extensively applied in cell biology and at a concentration of up to $40 \%(\mathrm{w} / \mathrm{w})$, DMSO-water mixtures are commonly employed in cell, tissue and organ cryopreservation [1]. DMSO also protects living systems against ionizing damage [2]. It is renowned for its ability to increase permeability across biomembranes [3]. DMSO was earlier recognised as a carrier of small molecules through a variety of biological barriers [4]. However controversial, its use and research has been extended not only to the penetration-enhancer excipients field but as an active pharmaceutical agent [4]. Current use of DMSO in dermatology is very limited practically as a solvent of 5 -aminolaevulinic acid in photodynamic therapy in non-melanoma skin cancers $[5,6]$.

In oncology, topical (pure) DMSO has been used in the treatment of local complications of vesicant cytotoxic extravasation, which include tissue necrosis and skin ulceration $[7,8]$. The mechanism of action of DMSO is based on free radical scavenging apart from being a skin enhancer, increasing the skin permeability and diffusion through the stratum corneum by the distortion of the lipid membranes, and its consequent extraction, and by displacement of bound water from keratin $[9,10]$. The limited use of DMSO in commercial topical formulations is due to the high quantities needed for skin enhancer action and a slow reversal of the lipid disruption [10]. Reported side effects include a mild burning at the application site and garlic breath odor [9]. DMSO is also used for the treatment of decubitus ulcers as an anti-inflammatory active agent [4].

Semisolid preparations are advantageous in topical application as they prolong the formulation period of contact with skin, reducing the number of applications. The DMSO interaction with the ceramide bilayers in particular with ceramide 2 (the predominant ceramide in skin lipids) has been investigated confirming experimental evidence that high concentrations of DMSO fluidize the stratum corneum lipids and enhance permeability [11]. This and many other studies have

*Corresponding author: Andreia Ascenso, iMed.ULisboa, Faculdade de Farmácia, Universidade de Lisboa, Lisbon, Portugal, Tel: +351926984823; E-mail: andreiaascenso@ff.ulisboa.pt

Received March 26, 2016; Accepted April 15, 2016; Published April 20, 2016

Citation: Simões S, Mendes T, Eleutério C, Pinto P, Ascenso A (2016) Development and Characterization of a Novel Vesicular System for Topical Delivery: An Opportunity for Dimethylsulfoxide. Med chem (Los Angeles) 6: 250256. doi:10.4172/2161-0444.1000354

Copyright: ( 92016 Simões S, et al. This is an open-access article distributed under the terms of the Creative Commons Attribution License, which permits unrestricted use, distribution, and reproduction in any medium, provided the original author and source are credited. 
investigated the interaction of DMSO and model membranes but little information exists about skin damage after topical application. A histopathological study from 1988 showed the topical application of $100 \%$ DMSO to the skin of nude mice causing severe skin irritation [12]. However, formulations containing DMSO are not abundant and when they exist they do not provide any insight on DMSO effect on the skin [13]. The presence of DMSO in a topical formulation may ensure simultaneously drug solubility and skin permeation.

The introduction of special vesicles, which are deformable enough to penetrate even narrow pathways between cells in the skin, started a new chapter in the dermal and transdermal delivery field [14]. Transfersomes (a trademark of IDEA, AG) composed of highly flexible membranes, were the first to introduce this concept [15]. These carriers comprise at least phosphatidylcholine and an edge-active molecule acting as the membrane softener. The vesicles have typically $150 \pm 50$ $\mathrm{nm}$ to ensure enough deformability. Many other deformable vesicles have then been introduced comprising one or a combination of edgeactive molecules, most often skin penetration enhancers, as carriers for dermal or transdermal delivery.

The aim of this research work was to develop and characterize a new type of semisolid formulation composed by deformable vesicles containing DMSO for topical application. To the best of our knowledge, soybean phosphatidylcholine (SPC)-DMSO nanovesicles have never been studied. In this study, tretinoin was used as the model substance.

\section{Materials and Methods}

\section{Materials}

SPC (S 100) was purchased from Lipoid ${ }^{\circledR}$ (Germany). DMSO $\geq$ 99.7\%, purity was acquired from Sigma-Aldrich ${ }^{\circledR}$ (UK). Tretinoin was purchased from Fagron ${ }^{\star}$ (Spain). Citric Acid 1-hydrate was purchased from Panreac Quimica, $\mathrm{SA}^{\star}$ (Spain). Ultrapure water was obtained in a MILLI-Q System from Millipore (USA). Dulbecco's modified Eagle's medium plus GlutaMAX ${ }^{\mathrm{ra}}$ dipeptide, PenStrep (Penicillin, Streptomycin), heat inactivated Fetal Bovin Serum and TrypLE $^{\mathrm{TM}}$ Express were purchased from Gibco ${ }^{\circ}$ by Life Technologies (UK). Phenazine methosulfate (PMS) was acquired from Applichem ${ }^{\circ}$ (Germany) and MTS Reagent Powder from Promega ${ }^{\circledR}$ (USA).

\section{Tretinoin loaded SPC-DMSO vesicles preparation}

Nanovesicles were prepared by adding Tretinoin $(0.05 \% \mathrm{~m} / \mathrm{m})$ to SPC $(20 \% \mathrm{~m} / \mathrm{m})$, DMSO $(30 \% \mathrm{~m} / \mathrm{m})$ and Citrate Buffer $50 \mathrm{mM}$ pH 5 (q.s. ad $100 \% \mathrm{~m} / \mathrm{m}$ ). This mixture was stirred for 2 hours at room temperature protected from light and air exposure. At the end, the formulation was filtered through 400 and $100 \mathrm{~nm}$ pore size polycarbonate membranes (Millipore ${ }^{\oplus}$, USA) under nitrogen stream in a filtration unit to obtain a vesicles homogenous size of $150 \pm 50$ $\mathrm{nm}$. In addition, the control formulation (without Tretinoin) was also prepared.

\section{Size and zeta potential}

The mean vesicle size and PDI were determined by dynamic light scattering (Malvern Zetasizer Nano-S, Malvern instruments ${ }^{\circledR}$, UK). PDI less than 0.2 indicates a homogenous and monodispersed population. Zeta Potential was determined by laser-doppler anemometry (Malvern Zetasizer Nano-Z, Malvern instruments ${ }^{\circ}$, UK) with the same samples used for size and PDI measurements. For these assays $10 \mu \mathrm{L}$ of the formulation were diluted in $4.5 \mathrm{~mL}$ of purified water.

\section{Tretinoin detection and quantification}

An HPLC system consisting of a 32 Karat Software (Beckam
Instruments ${ }^{\oplus}$, USA), a Midas Spark 1.1 autoinjector (Spark ${ }^{\oplus}$, Netherlands) $^{\circ}$ and a Diode-Array 168 detector (Beckman Instruments ${ }^{\oplus}$, USA) was used for tretinoin quantification. Chromatographic separations were performed using a reversed phase chromatography column (Lichrocart 250-4, $5 \mu \mathrm{m} \mathrm{C18,} 250 \mathrm{~mm} \times 4 \mathrm{~mm}$, Merck Millipore ${ }^{\oplus}$, Germany). The detection wavelength was $342 \mathrm{~nm}$ and the mobile phase was composed of $0.01 \%$ trifluoroacetic acid and acetonitrile $(15: 85, \mathrm{v} / \mathrm{v}, \%)$ with a flow rate of $1.0 \mathrm{~mL} / \mathrm{min}$ and an injection volume of $50 \mu \mathrm{L}$. This methodology was previously validated as reported in literature [16].

\section{Tretinoin yield}

Drug yield (equation 1) was determined after separation and removal of the active non-incorporated fraction: the formulation (1 $\mathrm{mL}$ ) was diluted in Citrate Buffer $50 \mathrm{mM} \mathrm{pH} 5$ and then centrifuged at $180000 \mathrm{~g}$ at $15^{\circ} \mathrm{C}$ during $2 \mathrm{~h}$ (Beckman Optima XL-90 Ultracentrifuge, GMI, USA). After centrifugation, the supernatant was removed and the precipitate was suspended in Citrate Buffer $50 \mathrm{mM} \mathrm{pH}$ 5. The active quantification was done by the HPLC method described above.

$$
\operatorname{Drug} \text { Yield }(\%)=\frac{[\text { Active }]_{\text {After centrifugation }}}{[\text { Active }]_{\text {Before centrifugation }}} \times 100
$$

\section{pH determination}

The $\mathrm{pH}$ was determined using a LLViscotrode combined glass electrode from Metrohm (Germany) and a $713 \mathrm{pH}$ meter from Metrohm (Germany). Measurements were made in triplicate.

\section{Transmission Electron Microscopy (TEM)}

TEM imaging was obtained using the electron microscope $\mathrm{H}-8100$ from Hitachi (Hitachi, Japan). The sample preparation consisted of placing a drop of vesicle diluted sample on top of a microscopic coppercoated grid (TEM grid Support Films of Formvar/Carbon, 200 mesh $\mathrm{Cu}$ ), letting it dry completely. After drying completely, $1 \%$ aqueous solution of phosphotungstic acid (Sigma, USA) was added for negative staining, waiting $45 \mathrm{~s}$ and wiping off the excess contrast with filter paper. The specimen was then viewed under the microscope with an accelerating voltage of $75 \mathrm{kV}$ and at different magnifications.

\section{Rheological analysis}

The apparent viscosity was determined at $20.4^{\circ} \mathrm{C}$ by using a Digital Brookfield Viscometer (Brookfield, DV-II, USA) with a spindle number 27 at $0.61,1.22,3.05,6.12,12.24,24.47,61.18$ and $122.36 \mathrm{~s}^{-1}$ for rheogram construction (equation 2). Readings were taken after 30 seconds.

$$
\text { Shear stress }=\text { Shear rate } \mathrm{x} \text { Viscosity }
$$

\section{Stability-on-storage}

Tretinoin-loaded SPC-DMSO nanovesicle formulations were stored at $4^{\circ} \mathrm{C}, 25^{\circ} \mathrm{C}$ (room temperature) and $40^{\circ} \mathrm{C}$, protected from light and air exposure. Samples were collected on days 7, 14, 28, 56, 12 and 180 for physical and chemical evaluation. The physical stability was evaluated by measuring size, PDI and Zeta Potential and chemical stability was evaluated through quantifying the amount of tretinoin remaining in the formulation by HPLC assay. The quantitative amount after fresh preparations (day 0) was normalized to $100 \%$.

\section{Deformability test}

Pressure driven transport of vesicle suspension (diluted with water to the final concentration of $2 \%(\mathrm{w} / \mathrm{v})$ SPC) through a microporous filter (PCTE) with $30 \mathrm{~nm}$ pore diameter was driven by an external pressure $(1.0 \mathrm{MPa})$, created by a nitrogen stream, and measured as a function of 
time. These measurements were made with a $1 \mathrm{~mL}$-filtration unit that miniaturizes the commercial available pressure filtration device (homebuilt). The suspension was collected into a container on a Sartorius LA620P scale (Sartorius, Germany) to determine automatically the weight of filtered suspension. Data was collected with the Wedge software for Windows (TAL Technologies Inc., USA). The average particle size was determined by dynamic light scattering, before and after the filtration experiment.

\section{DSC analysis}

DSC measurements were performed using a calorimeter DSC Q-200 model (Texas Instruments, USA). Indium was used for calibration $\left(\mathrm{Tm}=430.61 \mathrm{~K} ; \Delta\right.$ Hfus $\left.=28.71 \mathrm{Jg}^{-1}\right)$. Lyophilised samples of empty and Tretinoin loaded SPC-DMSO vesicles were weighed $(\sim 10$ $\mathrm{mg}$ ) on aluminium pans which were sealed under air. Nitrogen was used as a purging gas and the scans of modulated DSC were performed in a range of $-50 /+50^{\circ} \mathrm{C}$ at a heating rate of $3^{\circ} \mathrm{C} / \mathrm{min}$.

\section{Cell viability}

The cytotoxicity potential of SPC-DMSO nanovesicles was evaluated in HaCat cells obtained from ATCC (USA). HaCat cells were grown in Dulbecco's modified Eagle's medium (DMEM) plus GlutaMAX ${ }^{\text {ma }}$ dipeptide (L-glutamine and L-alanine), rich in glucose, supplemented with $10 \%$ fetal bovine serum (FBS), $1 \%$ penicillin-streptomycin $(10,000$ $\mathrm{U} / \mathrm{mL}$ ) and incubated at $37^{\circ} \mathrm{C}$ in a humidified atmosphere with $5 \%$ $\mathrm{CO}_{2}$. HaCat cells were exposed for $24 \mathrm{~h}$ to the different concentrations of SPC-DMSO nanovesicles formulations previously sterilized by filtration $(0.2 \mu \mathrm{m})$. Formulations containing SPC, DMSO and Tetinoin were tested in the proportion 10:15:0.025 (w:w:w). At the end of the exposure time, the formulation diluted in culture medium was removed and cells were gently washed with DMEM without supplementation, to eliminate any interferences [17]. Then, PMS+MTS DMEM solution was added and incubated at $37^{\circ} \mathrm{C}$ for $2 \mathrm{~h}$. Absorbance was measured at $490 \mathrm{~nm}$ (Microplate Reader, BioTek ${ }^{\star}$ Instruments, USA). Besides the negative control (non-exposed cells), results were also compared to SPC-DMSO empty nanovesicles (without Tretinoin), DMSO in Citrate Buffer $50 \mathrm{mM} \mathrm{pH} \mathrm{5,} \mathrm{solution} \mathrm{and} \mathrm{SPC} \mathrm{vesicles} \mathrm{made} \mathrm{by} \mathrm{dispersion} \mathrm{in}$ Citrate Buffer $50 \mathrm{mM} \mathrm{pH} 5$ (without DMSO).

\section{In vivo skin application and histological assessment}

The effect of DMSO, empty SPC-DMSO vesicles and of tretinoinloaded SPC-DMSO vesicles was compared when epicutaneously applied to back skin of shaved BALB/c female mice (20-25 g). Animals were housed in separate polypropylene cages of suitable size and kept under standardized environmental conditions throughout the study. Mice were divided into three groups of three animals each and treated daily by topically applying to the back $\left(6 \mathrm{~cm}^{2}\right)$, for 10 days, $100 \mu$ lof: group (II) DMSO; group (III) empty SPC-DMSO vesicles and group (IV) $0.05 \%$ tretinoin loaded SPC-DMSO vesicles. Animals were examined for signs of erythema each day before treatment throughout the treatment period and given an overall "irritation score" defined by Draize et al.: $0=$ no differences, $1=$ light erythema, $2=$ well defined erythema, $3=$ strong erythema, and $4=$ very strong erythema with presence of a scar [18]. At the end of tenth day, the animals were sacrificed. Skin biopsies (1 $\mathrm{cm}^{2}$ ) were taken from all treated areas, preserved in $10 \%$ formalin solution and processed for histopathological studies. Hematoxylin and eosin were used for staining the sections. Finally, the specimens were observed under a high power light microscope (Olympus, AH-2 Vanox, Japan). All animal experiments were carried out with the permission of the local animal ethical committee in accordance with the EU Directive (2010/63/EU), Portuguese law (DL 113/2013) and all relevant legislation. The experimental protocol was approved by Direcção Geral de Alimentação e Veterinária.

\section{Statistical analysis}

The results were reported as mean \pm standard deviation (SD) of at least three measurements. The results were statistically analysed by Analysis of Variance (ANOVA), for many group comparisons, while Student's t-test was applied for comparison between two samples, using SigmaPlot 12.0 software. The differences were considered statistically significant when $\mathrm{p}<0.05$.

\section{Results and Discussion}

\section{Vesicle size, superficial charge, encapsulation parameters and pH determination}

A novel nanovesicle was developed, consisting of three main components, SPC, DMSO and a buffer solution to guarantee final $\mathrm{pH}$ compatible with skin application [19]. This very simple semisolid formulation is easy to prepare, cold processed, and permitted to incorporate $30 \%$ of a lipid fluidizing component, DMSO, in a lipid vesicular organization (Figure 1). Vesicles presented a size within the expected range $(150 \pm 50 \mathrm{~nm})$ and a Polydispersity Index (PDI) below 0.2 . Zeta potential was negative. Besides, Tretinoin leads to a slightly modular increase in the superficial vesicle charge. Tretinoin Yield was high as theoretically expected (Table 1 ). This may be due to the lipophilicity of Tretinoin (Log P of $6.83 \pm 0.36$ ) and DMSO solubility that allows the incorporation mainly into the vesicle membrane. This result was consistent and relatively higher compared to the results described in the literature for Tretinoin loaded liposomes, ethosomes, niosomes, and other deformable vesicle formulations [20].

\section{Visualization of vesicles by electron microscopy}

To complete the characterization of the vesicles, SPC-DMSO vesicle preparation was examined by negative stain Transmission Electron Microscopy. Vesicles appeared as multilamellar vesicles. The lamellae of the vesicles were evenly spaced to the core (Figure 1 ). The images resemble those obtained for other liposomal systems [21]. DMSO interaction with model membranes is widely studied; however, vesicles containing DMSO have never been studied and visualized.

\section{Rheological analysis}

SPC-DMSO vesicles formulation exhibited a non-newtonian time-dependent behaviour type of pseudoplastic flow (Figure 2) and the apparent viscosity decreases with the shear rate increase. DMSO interaction with the phospholipid bilayer led to a reduction of the energy required for bilayer deformation. Therefore, with the shear rate increase damaged vesicles are quickly reconstituted causing a decrease in the apparent viscosity [22]. As a result, this formulation should have a good spreadability, and it would maintain contact on the application site long enough for the drug to exert its pharmacological effect.

\section{Stability-on-storage}

SPC-DMSO vesicles were stored at different temperatures. Concerning the vesicle size, a slight increase around day 120 after preparation was observed for all tested temperatures, even though it was still within the expected range $(150 \pm 50 \mathrm{~nm}$, approximately 175 $\mathrm{nm}$ ) (Figure 3A). Therefore, these storage conditions did not influence the vesicle bilayer as vesicles maintained their size and structure. This result was consistent with another monitored study of tretinoin loaded-penetration enhancer containing vesicles, during 90 -days at 4 $\pm 1^{\circ} \mathrm{C}$ [22]. The PDI was lower than 0.2 during the evaluation study and for all temperatures studied (data not shown). These results were associated to a homogenous vesicle size. Regarding the Zeta Potential, a vesicle surface negative charge (around $-20 \mathrm{mV}$ ) was obtained as expected (data not shown). Negative surface charge will prevent vesicle 


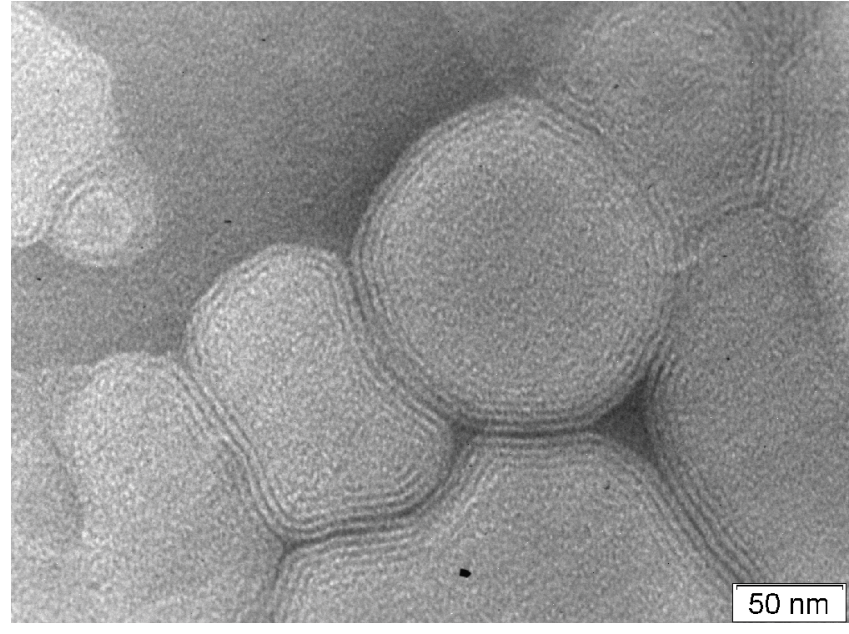

Figure 1: Microscopic appearance of SPC-DMSO formulations obtained by transmission electron microscopy.

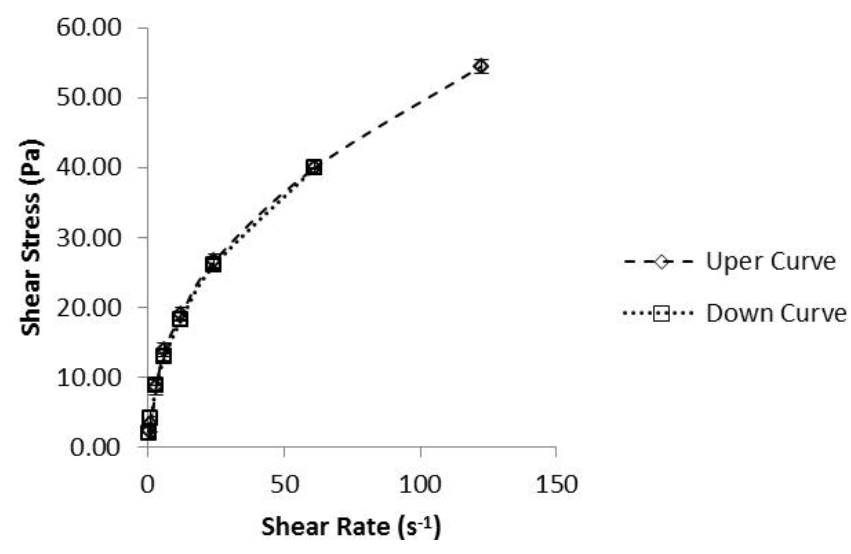

Figure 2: Rheogram of SPC-DMSO vesicle formulation (results expressed as mean \pm SD performed in triplicate).

fusion and aggregation thus maintaining the vesicle mean diameter constant (Figure 3A). SPC has a zwitterionic behaviour, and on the other hand, the acidic $\mathrm{pH}$ could also affect both charges of Tretinoin (pKa) and DMSO in formulation [20]. During the time under study, an increased modular zeta potential value was observed at $40^{\circ} \mathrm{C}$ that could be explained by a higher interaction in the vesicle membrane affected by temperature. The Tretinoin Yield remained high $(>80 \%)$ during the storage time course for all tested temperatures, probably due to the Tretinoin stabilisation in the vesicle membrane (Figure 3B).

\section{Deformability test}

Vesicle flux is a measure of vesicle deformability. Tretinoin loaded SPC-DMSO vesicles and empty ones were forced to pass through pores smaller than their own size (6 times). Flux was higher for Tretinoin loaded SPC-DMSO vesicles compared to the empty ones (Figure $4 \mathrm{~A})$. This indicates a greater deformability degree with the Tretinoin incorporation that could be explained by the possible interaction of this active and DMSO in the lipid layer structure. However, during the pressure driven transport, vesicles reduced their diameter equally by $35 \%$ (Figure $4 \mathrm{~B}$ ). These findings are in accordance with the size reduction for other deformable vesicles [23].

\section{Differential Scanning Calorimetry (DSC) analysis}

Empty SPC-DMSO vesicles and Tretinoin-loaded SPC-DMSO
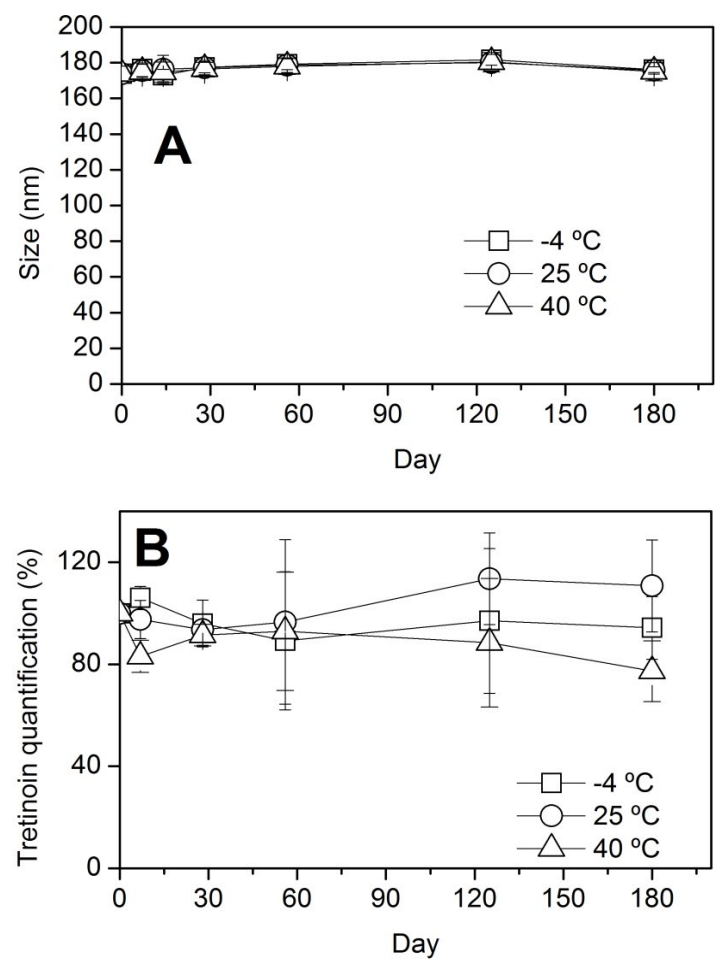

Figure 3: Physical and chemical evaluation of Tretinoin loaded SPC-DMSO nanovesicles during 180 days. (A) Size $(\mathrm{nm})$; (B) Tretinoin Quantification (\%) (results expressed as mean \pm SD of three independent batches).

vesicles, both in lyophilised form, showed a similar enthalpy $(\simeq 12 \mathrm{~J} / \mathrm{g})$ due to the presence of the same lipid amount $(20 \%)$. However, a shift of the transition temperature from $-11.76^{\circ} \mathrm{C}$ to $-9.77^{\circ} \mathrm{C}$ was observed which may suggest the incorporation and interaction of Tretinoin with vesicle components, mainly the lipid membrane. As expected, empty vesicles have a transition temperature closer to $-20^{\circ} \mathrm{C}$, the theoretical value of pure SPC (according to the manufacturer's specification). Tretinoin showed a melting peak corresponding to literature description $\left(\Delta \mathrm{H}=92 \mathrm{~J} / \mathrm{g}\right.$; Maximum peak temperature $\left.=184^{\circ} \mathrm{C}\right)[22]$.

\section{Cell viability analysis}

Microscopic observation $24 \mathrm{~h}$ after HaCat cells exposure to SPCDMSO vesicles, SPC vesicles and DMSO buffer solutions (Figures S1, S2 and S3), respectively, showed a more pronounced decrease of cell number and morphological alterations for the higher concentrations, compared to the negative control. SPC exposure promoted a morphological alteration to a rounder and more swollen conformation. This may be due to the interference of SPC in the sphingolipid metabolism that inhibits the cell proliferation and induces apoptosis [24]. Cell exposure to DMSO produced drastic morphological alterations in HaCat cells probably by the cytotoxic effects of DMSO on the tested concentrations. At lower concentrations a lower impact on cell morphology was obtained. This result was consistent with an MTS assay in HaCat cell line with $1.5 \%(\mathrm{v} / \mathrm{v})$ of DMSO which did not induce a decrease in cell viability [25]. Comparing the microscopic figures, there are more similarities between the cells previously exposed to SPC-DMSO vesicle formulation and DMSO buffer solution than those exposed to SPC vesicles.

The cell viability assay was consistent with the optical microscopic observations. There were only significant differences of cell viability for higher SPC concentrations ( $\geq 2.5 \%$ ), presenting only $20 \%$ of cell viability 
(Figure 5A). Tretinoin did not increase the formulation cytotoxicity at the tested concentrations (Figure $5 \mathrm{~B}$ ). The cell viability was about $40 \%$ only for SPC vesicles at $10 \%$ lipid concentration (data not shown). On the other hand, the DMSO cytotoxicity profile was very similar to the empty and Tretinoin loaded vesicles (data not shown). Therefore, DMSO dispersion showed a marked cell viability reduction only for the major concentrations $(\geq 3.75 \%)$. In this case, the formulation toxicity can be explained by the DMSO interference on the protective function of cell plasmatic membrane by the disturbance in the lipid bilayer [26].

\section{In vivo skin application of SPC-DMSO vesicles}

It is very difficult to have a correspondence between the in vitro toxicity in keratinocytes and the in vivo damage of chemical agents applied on the skin. Even considering the numerous models of skin lipid barrier, a definitive model which is either experimental or computational is still desirable [27]. DMSO is known to enhance the permeability of both hydrophobic and hydrophilic molecules and DMSO effect was found to be concentration dependent. DMSO is also used as a cryoprotectant. However, there is a great controversy on DMSO skin application regarding the reversibility of its effects on breaching the skin barrier [27]. In order to evaluate the effect of repeated application of new developed formulation, an in vivo assay was performed. We observed that these new vesicles produced no erythema by repeated application to the skin. Even considering the irritation potential of Tretinoin, the drug incorporation in such vesicles was able to reduce skin irritation, macroscopically (Figure 6A). As previously found, lipid-rich formulations are able to improve the skin tolerability to Tretinoin [28]. Histological analysis of skin (I) Untreated, (II) Treated with DMSO, (III) Treated with empty SPC-DMSO vesicles, and (IV) Treated with Tretinoin-loaded SPC-DMSO vesicles, presented no

\begin{tabular}{|c|c|c|c|c|c|}
\hline Formulations and Parameters & Size $(\mathrm{nm})$ & PDI $^{\mathbf{a}}$ & Zeta Potential (mV) & Tretinoin Yield (\%) & pH \\
\hline Empty SPC-DMSO vesicles & $182 \pm 1$ & $0.131 \pm 0.013$ & $-27.0 \pm 0.3$ & $N A^{b}$ & $6.3 \pm 0.03$ \\
\hline Tretinoin Loaded SPC-DMSO vesicles & $184 \pm 1$ & $0.169 \pm 0.022$ & $-30.2 \pm 1.0$ & $92.5 \pm 5.8$ & $6.5 \pm 0.04$ \\
\hline
\end{tabular}

aPolydispersity Index; ' $\mathrm{Not}$ applicable

Table 1: Characterization of SPC-DMSO vesicles. NA: Not Applicable (results expressed as mean \pm SD of three independent batches).

A

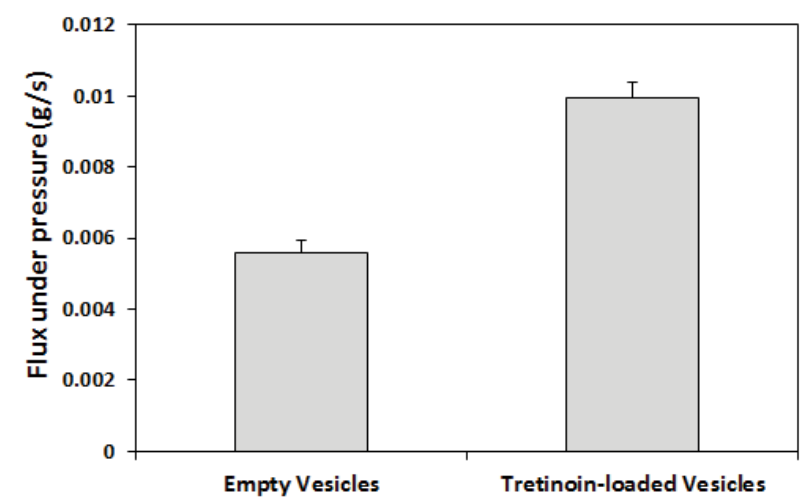

B

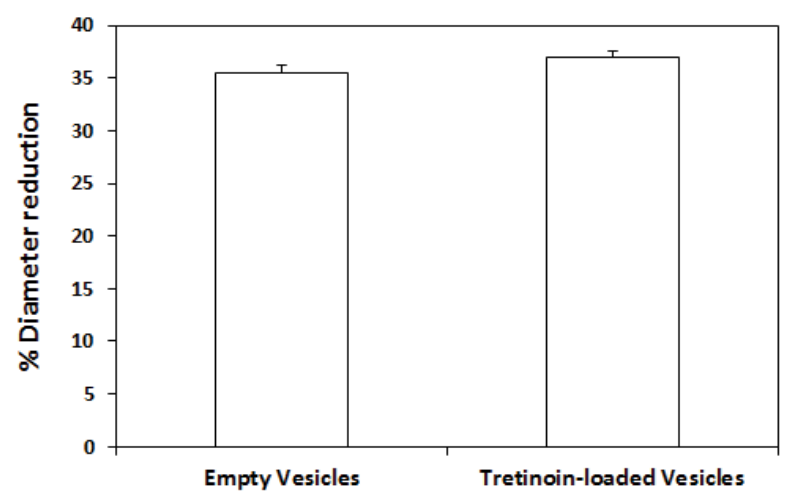

Figure 4: Pressure driven transport of Empty SPC-DMSO and Tretinoin loaded vesicles expressed as mean \pm SD ( $\mathrm{g} / \mathrm{s}$ ) of three independent batches. (A) Flux of the vesicles passing through $30 \mathrm{~nm}$ pores; (B) Diameter reduction of vesicles after the pressure driven transport assay. Data expressed as mean \pm SD (\%) of three independent batches. Statistical analysis: t-test, * ${ }^{*}$ eans significantly different $(p<0.05)$.
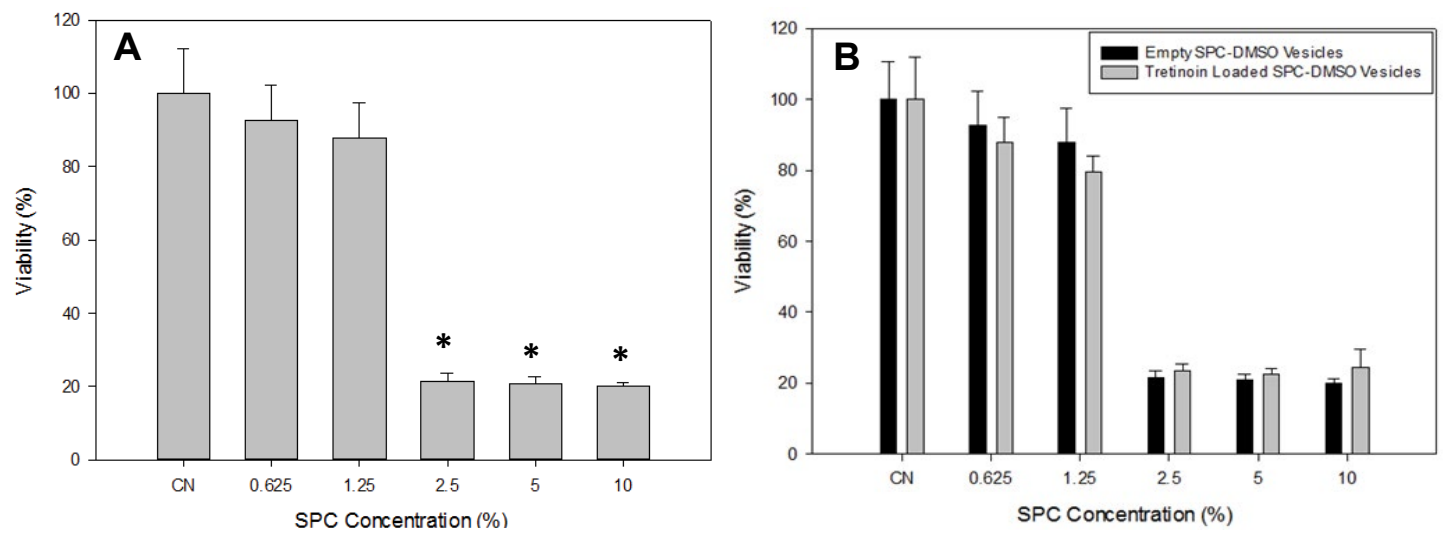

Figure 5: Cell viability expressed as mean $\pm \mathrm{SD}(\%), \mathrm{n}=12$ determined by MTS assay. (A) Empty SPC-DMSO vesicles compared to the negative control; (B) Empty and Tretinoin loaded SPC-DMSO vesicles. Statistical analysis: One Way Anova with All Pairwise (between empty and loaded vesicles and between different SPC or DMSO concentrations in each experiment) Multiple Comparisons and Multiple Comparisons versus Control Group (Tukey test), ${ }^{*}$ means statistically different $(p<0.05)$. 
A

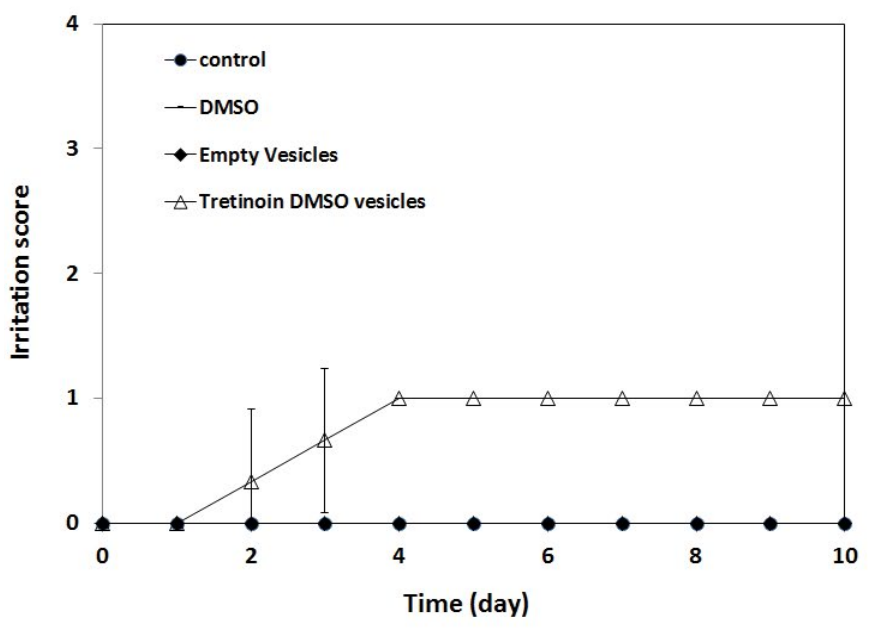

B
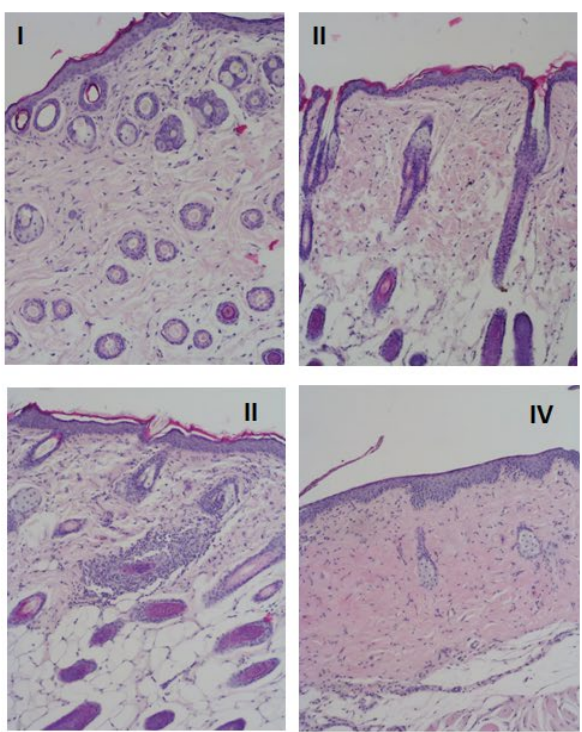

Figure 6: Skin Irritation Potential of skin (I) Untreated, (II) Treated with DMSO, (III) Treated with empty DMSO vesicles, and (IV) Treated with Tretinoin-loaded $\mathrm{SPC}$-DMSO vesicles in mice skin (mean $\pm \mathrm{SD}, \mathrm{n}=3$ for each formulation). (A) Irritation score: $0=$ no difference between the tretinoin-treated animals and control, $1=$ light erythema, $2=$ well defined erythema, $3=$ strong erythema, and $4=$ very strong erythema with presence of a scar. (B) Sections of mouse skin stained by hematoxylin-eosin standard procedure and observed by microscopy (original magnification 10X).

inflammatory signals (Figure 6B). Skin treated with Tretinoin vesicles (IV) presented some extent of epidermis hyperplasia as expected since Tretinoin mechanism of action is based on keratinocyte proliferation increase [29].

\section{Conclusion}

In summary, it was possible to develop and characterize a new type of deformable nanovesicle made of SPC and DMSO. With this study it was demonstrated that this new type of vesicles has advantages like high deformability and adequate rheology so that facilitated topical administration and skin penetration could be achieved. This formulation showed an excellent chemical and physical stability during 6 months at different temperatures as well as low cytotoxicity effect below $1.25 \%$ and $1.88 \%$ of SPC and DMSO, respectively. The possibility of reducing DMSO concentrations and frequency of its administration, and consequently, the side effects, would bring benefits to the therapy. This new formulation could also be a promising alternative to the intravenously and subcutaneous administered drugs used for the extravasation treatment of cytotoxic drugs. The use of a semisolid preparations containing DMSO could be advantageous as DMSO can remain in contact with the skin over an extended period of time. DMSO binds and neutralizes free radicals, stabilizes the cellular membranes, slows down or stops cell death in injured areas preventing or solving skin ulcer. The action of the substances associated to the vesicles can be limited on the damaged skin area. The proposed composition was able to confront the most challenging problem of skin irritation by DMSO and the data obtained with HaCat cells may in the future facilitate the work with other cell lines to complement the safety assessment of the developed formulation.

Therefore, SPC-DMSO vesicles offer a platform technology and may be extrapolated and explored for new applications due to their numerous advantages, and other actives might be incorporated for further pharmacological applications.

\section{Acknowledgements}

This work was partially funded by Fundação para a Ciência e Tecnologia (FCT, Portugal) through UID/DTP/04138/2013. We thank to Manuela Carvalheiro and Manuela Gaspar for cell culture provisions and Ana Salgado for technical assistance.

\section{References}

1. Lakey JR, Anderson TJ, Rajotte RV (2001) Novel approaches to cryopreservation of human pancreatic islets. Transplantation 72: 1005-1011.

2. Milligan JR, Ward JF (1994) Yield of single-strand breaks due to attack on DNA by scavenger-derived radicals. Radiat Res 137: 295-299.

3. Bonora S, Markarian SA, Trinchero A, Grigorian KR (2005) DSC study on the effect of dimethysulfoxide (DMSO) and diethylsulfoxide (DESO) on phospholipid liposomes. Thermochim Acta 433: 19-26.

4. Capriotti K, Capriotti JA (2012) Dimethyl sulfoxide: history, chemistry, and clinical utility in dermatology. J Clin Aesthet Dermatol 5: 24-26.

5. Christensen E, Skogvoll E, Viset T, Warloe T, Sundstrøm S (2009) Photodynamic therapy with 5-aminolaevulinic acid, dimethylsulfoxide and curettage in basal cell carcinoma: a 6-year clinical and histological follow-up. J Eur Acad Dermatol Venereol 23: 58-66.

6. Souza CS, Felicio LB, Ferreira J, Kurachi C, Bentley MV, Tedesco AC, et al. (2009) Long-term follow-up of topical 5-aminolaevulinic acid photodynamic therapy diode laser single session for non-melanoma skin cancer Photodiagnosis Photodyn Ther 6: 207-213.

7. Langer SW (2010) Extravasation of chemotherapy. Curr Oncol Rep 12: 242-246.

8. de Wit M, Ortner P, Lipp HP, Sehouli J, Untch M, et al. (2013) Management of cytotoxic extravasation - ASORS expert opinion for diagnosis, prevention and treatment. Onkologie 36: 127-135.

9. Schulmeister L (2010) Preventing and managing vesicant chemotherapy extravasations. J Support Oncol 8: 212-215.

10. Lane ME (2013) Skin penetration enhancers. Int J Pharm 447: 12-21.

11. Notman R, den Otter WK, Noro MG, Briels WJ, Anwar J (2007) The permeability enhancing mechanism of DMSO in ceramide bilayers simulated by molecular dynamics. Biophys J 93: 2056-2068.

12. Lashmar UT, Hadgraft J, Thomas N (1989) Topical application of penetration 
Citation: Simões S, Mendes T, Eleutério C, Pinto P, Ascenso A (2016) Development and Characterization of a Novel Vesicular System for Topical Delivery: An Opportunity for Dimethylsulfoxide. Med chem (Los Angeles) 6: 250-256. doi:10.4172/2161-0444.1000354

enhancers to the skin of nude mice: a histopathological study. J Pharm Pharmacol 41: 118-122.

13. Casiraghi A, Ardovino P, Minghetti P, Botta C, Gattini A, et al. (2007) Semisolid formulations containing dimethyl sulfoxide and alpha-tocopherol for the treatment of extravasation of antiblastic agents. Arch Dermatol Res 299: 201 207.

14. Ascenso A, Ribeiro HM, Marques HC, Simoes S (2011) Topical delivery of antioxidants. Curr Drug Deliv 8: 640-660.

15. Cevc G, Blume G (1992) Lipid vesicles penetrate into intact skin owing to the transdermal osmotic gradients and hydration force. Biochim Biophys Acta 1104: 226-232.

16. Tashtoush BM, Jacobson EL, Jacobson MK (2007) A rapid HPLC method for simultaneous determination of tretinoin and isotretinoin in dermatological formulations. J Pharm Biomed Anal 43: 859-864.

17. Huang KT, Chen YH, Walker AM (2004) Inaccuracies in MTS assays: majo distorting effects of medium, serum albumin, and fatty acids. Biotechniques 37 : $406,408,410-412$

18. Draize JH, Woodward G, Calvary HO (1944) Methods for the study of irritation and toxicity of substances applied topically to the skin and mucous membranes. J Pharmacol Exp Ther 82: 377-390.

19. Lambers H, Piessens S, Bloem A, Pronk H, Finkel P (2006) Natural skin surface $\mathrm{pH}$ is on average below 5 , which is beneficial for its resident flora. Int $\mathrm{J}$ Cosmet Sci 28: 359-370.

20. Ascenso A, Cruz M, Euletério C, Carvalho FA, Santos NC, et al. (2013) Novel tretinoin formulations: a drug-in-cyclodextrin-in-liposome approach. J Liposome Res 23: 211-219.
21. Touitou E, Dayan N, Bergelson L, Godin B, Eliaz M (2000) Ethosomes - novel vesicular carriers for enhanced delivery: characterization and skin penetration properties. J Control Release 65: 403-418.

22. Manconi M, Sinico C, Caddeo C, Vila AO, Valenti D, et al. (2011) Penetration enhancer containing vesicles as carriers for dermal delivery of tretinoin. Int $J$ Pharm 412: 37-46.

23. Ascenso A, Pinho S, Eleutério C, Praça FG, Bentley MV, et al. (2013) Lycopene from tomatoes: vesicular nanocarrier formulations for dermal delivery. J Agric Food Chem 61: 7284-7293.

24. Geilen CC, Wieder T, Orfanos CE (1997) Ceramide signalling: regulatory role in cell proliferation, differentiation and apoptosis in human epidermis. Arch Dermatol Res 289: 559-566.

25. Altenburger R, Kissel T (1999) The human keratinocyte cell line HaCaT: an in vitro cell culture model for keratinocyte testosterone metabolism. Pharm Res 16: 766-771.

26. Maupas C, Moulari B, Béduneau A, Lamprecht A, Pellequer Y (2011) Surfactant dependent toxicity of lipid nanocapsules in HaCaT cells. Int J Pharm 411: 136-141.

27. Notman R, Anwar J (2013) Breaching the skin barrier--insights from molecular simulation of model membranes. Adv Drug Deliv Rev 65: 237-250.

28. Ascenso A, Salgado A, Euletério C, Praça FG, Bentley MV, et al. (2014) In vitro and in vivo topical delivery studies of tretinoin-loaded ultradeformable vesicles. Eur J Pharm Biopharm 88: 48-55

29. Kligman AM, Grove GL, Hirose R, Leyden JJ (1986) Topical tretinoin for photoaged skin. J Am Acad Dermatol 15: 836-859. 